Document downloaded from:

http://hdl.handle.net/10251/120913

This paper must be cited as:

Rodríguez-Vallejo, M.; Montagud-Martínez, D.; Monsoriu Serra, JA.; Ferrando Martín, V.; Furlan, WD. (2018). Relative Peripheral Myopia Induced by Fractal Contact Lenses. Current Eye Research. 43(12):1514-1521. https://doi.org/10.1080/02713683.2018.1507043

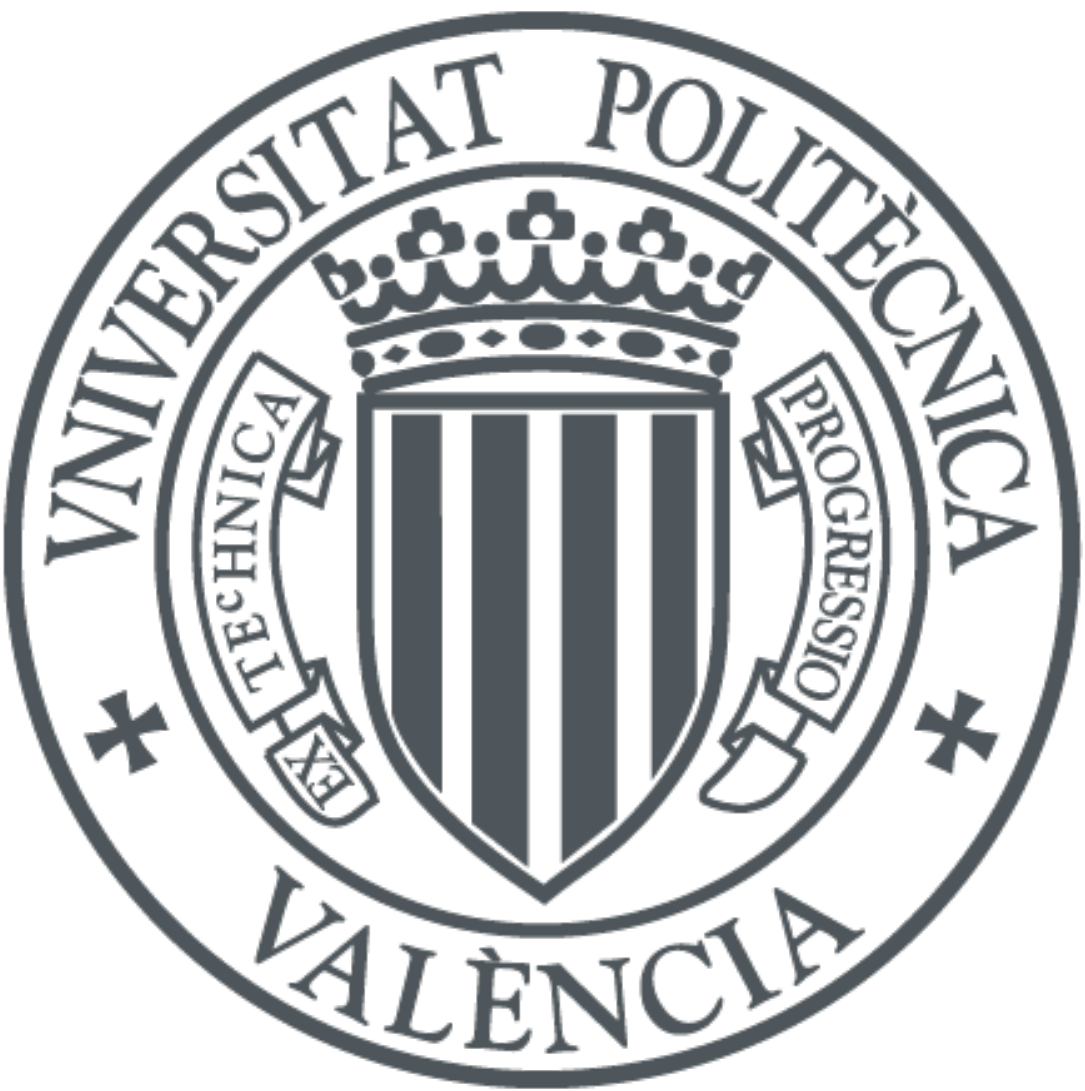

The final publication is available at

http://doi.org/ 10.1080/02713683.2018.1507043

Copyright Informa UK (Informa Healthcare)

Additional Information 


\section{Relative peripheral myopia induced by Fractal Contact Lenses}

Manuel Rodriguez-Vallejo ${ }^{1^{*}}$, Diego Montagud ${ }^{2}$ Juan A. Monsoriu $^{2}$, Vicente Ferrando ${ }^{3}$ and Walter D. Furlan ${ }^{3}$

${ }^{1}$ Department of Ophthalmology (Qvision), Vithas Virgen del Mar Hospital, 04120, Almería, Spain

${ }^{2}$ Centro de Tecnologías Físicas, Universitat Politècnica de València, 46022 Valencia, Spain

${ }^{3}$ Departamento de Óptica, Universitat de València, 46100 Burjassot, Spain

${ }^{*}$ Corresponding author: manuelrodriguezid@qvision.es 


\section{Abstract.}

\section{Purpose:}

To assess the peripheral refraction induced by Fractal Contact Lenses (FCLs) in myopic eyes by means of a two-dimensional Relative Peripheral Refractive Error (RPRE) map.

\section{Materials and Methods:}

This study involved twenty-six myopic subjects ranging from $-0.50 \mathrm{D}$ to $-7.00 \mathrm{D}$. FCLs prototypes were custom-manufactured and characterized. Corneal topographies were taken in order to assess correlations between corneal asphericity, lens decentration. Two-dimensional RPREs were measured with an open-field autorefractor at 67 points, covering the central 60 $\mathrm{x} 30$ degrees of the visual field. The bidimensional RPRE vector components: $\mathrm{M}, \mathrm{J}_{0}$ and $\mathrm{J}_{45}$ of the difference between the values obtained with and without the FCLs in the eye were obtained. Additionally, the FCL induced peripheral refraction in tangential and sagittal planes was computed along the horizontal meridian.

\section{Results:}

Significant correlations were found between the corneal asphericity and vector components of the RPRE in the nacked eyes. FCLs were decentered a mean of $0.7 \pm 0.19 \mathrm{~mm}$ to the temporal cornea. $\mathrm{M}$ decreased asymmetrically between nasal and temporal retina after fitting the FCLs with a significant increment of the myopic shift beyond $10^{\circ}(\mathrm{p}<0.05)$. The maximum myopic shift at the peripheral retina $(M=-1.3 \mathrm{D})$ was located at $20^{\circ}$. The twodimensional RPRE maps showed the FCLs decentration. Induced by the FCLs, significant differences for all vector components were found in peripheral retina.

\section{Conclusions}

As predicted by ray-tracing simulations, FCLs fitted in myopic eyes produce a myopic shift of the RPRE. The two-dimensional RPRE maps show information about the lens performance that is hidden in the conventional one-dimensional meridional representations.

Key Words: myopia progression, contact lenses, fractal, peripheral refractive error, twodimensional maps. 


\section{Introduction}

During the last years myopia control therapies have deserved a large interest among researchers and vision care professionals. Several methods have been proposed to slow myopia progression; among them, non-pharmacological treatments, like orthokeratology and peripheral defocus modifying contact lenses (CLs) achieved very good outcomes, which has been attributed to the induction of a myopic Relative Peripheral Refractive Error (RPRE), although the optical mechanisms for myopia development still remain controversial. ${ }^{1-7}$ Different designs of multifocal CLs were proposed to this aim, ${ }^{6,8-10}$ and consequently, the amount and extension of the induced RPRE vary among lenses. Since the ocular growth during the emmetropization process might depend on the stimulated retinal areas, ${ }^{11}$ it is expected that different designs will provide different results. Therefore, to obtain information about RPRE induced by different lenses in the spherical, but also in the astigmatic component of the refraction, across the whole paracentral retina seems to be of great importance to understand the lens success in myopia progression. In particular, it is likely that the astigmatic component of the RPRE plays a role in the myopia development in humans, as it has been demonstrated it plays in the emmetropization in monkeys ${ }^{12}$ and chicks. ${ }^{12-13}$

In a previous paper, ${ }^{10}$ we have proposed a new design of CLs for myopia control, named Fractal Contact Lenses (FCLs). The potentiality of FCLs to produce a myopic RPRE was demonstrated by ray tracing methods in model eyes. However, the good theoretical performance obtained with FCLs has not been still validated in real eyes. Therefore, the main aim of this study is to assess the peripheral refraction induced by FCLs. To do that, FCLs prototypes were specially manufactured and characterized. In order to obtain a complete assessment of the lens performance in real myopic eyes, we measured the RPRE induced by the lenses at 67 locations 
across the $60^{\circ} \times 30^{\circ}$ of right eye visual field with an open field autorefractometer. The mean values of the measured dioptric power vectors $\left(\mathrm{M}, \mathrm{J}_{0}\right.$ and $\left.\mathrm{J}_{45}\right)$, were represented as contour plots in 2D power maps. In this sense, our approach improves the assessment methods employed in previous works evaluating contact lenses, because we are able to obtain complete 2D information about the induced RPRE at different positions of the visual field.

\section{Materials and Methods}

\section{Contact Lenses construction and characterization}

FCLs are multizone bifocals with a special design as described in Ref. [10]. The profile of the lenses are shown in Fig. 1A). A set of FCLs prototypes with treatment labeled power of +2.00 D and correction powers ranging from $-0.50 \mathrm{D}$ to $-7.00 \mathrm{D}$ in $-0.50 \mathrm{D}$ steps, were fabricated using a precision lathe (Optoform 40, Sterling Ultra Precision, Largo, USA). The lens material was Hioxifilcon A (Benz G5X p-GMA/HEMA), whose refractive index, hydrated and at $35^{\circ}$, is 1.401. Each FCLs prototype was manufactured with a diameter of $14.50 \mathrm{~mm}$ and two different base curves: $8.4 \mathrm{~mm}$ and $8.6 \mathrm{~mm}$. The constructed lenses were assessed with the Nimo TR1504 (LAMBDA-X, Nivelles, Belgium) contact lens power mapper (version 4.2.6.0 r477). The instrument software allows to obtain the dioptric power profiles of multifocal CLs, however it is not able to locate the zones in a multizone CLs and it is the operator who must enter the radii of the zones (with a maximum of five zones). For this reason, we developed a custom software using MATLAB (Mathworks, Inc., Natwick, MA) to precisely detect the power transition

between therapeutic and compensation zones. ${ }^{14}$ The algorithm computes the second derivative of the dioptric power profile exported from the NIMO, and shows the transition between two zones 
of different power (see Fig. 1B). The program also obtains the parabolic profiles of both the base (far distance) power of the lens and its therapeutic power, see Fig. 1C). For a given radius $\rho$ of the pupil, the fourth-order Zernike spherical aberration of the lens: $\mathrm{C}_{4,0}$ is obtained by fitting the profile to a parabolic curve $\mathrm{P}(\mathrm{r})=\mathrm{P}_{0}+\mathrm{br}^{2}$ as: $\mathrm{C}_{4,0}=\mathrm{b} \rho^{4} / 24 \sqrt{5}$ where $\mathrm{P}_{0}$ is the paraxial power (at r=0) (see Fig. 1C). ${ }^{15}$ The power profile at the therapeutic zones is obtained by substracting the results of Fig. 1B and 1C) as shown in Fig. 1D). By using this method, each lens was relabelled with its true (experimental) therapeutic and correction power.

\section{Subjects and procedures}

The research adhered to the tenets of the Declaration of Helsinki. All participants gave written consent after explanation of the nature of the study which was approved by the Institutional Review Board of the University of Valencia. Twenty-six myopic subjects (mean age $23.77 \pm$ 3.62 years) participated in this study (18 females and 8 males). All participants underwent a complete eye exam including objective and subjective refraction and slit-lamp exploration. Only right eyes were considered. Inclusion criteria were: myopic eyes ranging from $-0.50 \mathrm{D}$ to $-7.00 \mathrm{D}$ (mean $-2.62 \pm 1.59 \mathrm{D}$ ) and astigmatism $\leq 0.75 \mathrm{D}$ with no ocular diseases, strabismus or amblyopia with normal and corrected distance visual acuity better than 0.2 log MAR.

Before fitting the FCLs, corneal topographies were taken for the naked eye with the Keratron Scout topographer (Optikon 2000 SpA, Rome, Italy). Elevation data were exported in binary format (.XLB and .ZLB extension files) and a custom software was programmed in MATLAB in order to compute corneal asphericities, fitting elevation data to a conic function, ${ }^{16}$ at the nasal and temporal sides, along the horizontal $\left(0^{\circ}-180^{\circ}\right)$ meridian. 
Subjects were fitted with the FCL of the constructed set having the compensation power closer to the spectacle refraction after correction of vertex distance power, and with base curve that best matched the corneal radius. The behaviour of the lenses, movement and centration, were evaluated by the examiner twenty minutes after fitting. Then corneal topographies were taken again but with the patient wearing the best fitted FCL. The distance from the centre of the first therapeutic zone and the centre of the pupil diameter was measured with the caliper tool of the Keratron Scout software to obtain the FCL decentration in each case.

\section{Peripheral Refractive Error}

Objective central and peripheral refractions were measured with an open-field autorefractor (Grand-Seiko WAM-5500, Grand-Seiko Co., Ltd., Hiroshima, Japan). All measurements were made in non-cyclopegic conditions at environmental mesopic light conditions to ensure minimum pupil diameters of $4 \mathrm{~mm}$ in all participants.

Participants looked at 67 fixation targets (high contrast circles of 1" diameter located on a $2 \mathrm{~m}$ distant wall) covering $60^{\circ} \times 30^{\circ}$ of the central visual field, see Fig. 2. Measurements were taken with the eye rotation technique, ${ }^{17}$ and the alignment was achieved with the instrument alignment camera, so that the pupil of the tested eye was centered with respect of the measuring axis. Participants fixated the targets sequentially from left to right, line by line from the top. Refractions in clinical notation (sphere, cylinder and axis) were exported for analysis in MATLAB code. Each measure was converted to vector components: spherical equivalent M, with/against the rule astigmatism: $\mathrm{J}_{0}$, and oblique astigmatism: $\mathrm{J}_{45}{ }^{18}$ The program requests the examiner to obtain a minimum of three averaged measures per fixation point up to having standard deviations lower than $0.3 \mathrm{D}$, to compute the mean values of $\mathrm{M}$, $\mathrm{J}_{0}$, ans $\mathrm{J}_{45}$. The RPRE 
was measured in each subject first with the naked eye (baseline state) and then in the same eye fitted with the FCL. The RPREs were calculated by substracting the central values of each vector component from the corresponding peripheral one. Contour maps representing the mean values of $\mathrm{M}, \mathrm{J}_{0}$ and $\mathrm{J}_{45}$ at each visual field location were generated using cubic interpolation of splines in steps of 0.5 degrees. Recorded data was used to compute also the tangential $\left(\mathrm{F}_{\mathrm{T}}=\mathrm{M}+\mathrm{J}_{0}\right)$ and sagittal $\left(\mathrm{F}_{\mathrm{S}}=\mathrm{M}-\mathrm{J}_{0}\right)$ power errors along the horizontal meridian.

\section{Statistical Analysis}

Statistical analysis was done using SPSS software version 20 (SPSS Inc., Chicago, IL, USA), and $\mathrm{p}<0.05$ was considered to indicate significance. Normal distributions were tested with the Shapiro-Wilk test. Paired t-tests were used to analyze the differences between the RPRE vector components obtained with the FCLs and with the nacked eye. Pearson correlation analyses were performed to determine the relationship between variables. Power analysis was performed using G Power version 3.1.9.2 (available at http://www.gpower.hhu.de/). The sample size in this study offered $88 \%$ statistical power at a 5\% level to detect a difference in RPRE of $0.25 \mathrm{D}$ with and without FCLs when the expected standard deviation (SD) of the mean difference was 0.44 D.

\section{Results}

\section{Contact Lenses: Power profiles and fitting}

The power profiles of the therapeutic zones of the constructed lenses had a mean value of $1.32 \pm$ $0.28 \mathrm{D}$, which resulted $0.68 \mathrm{D}$ lower than the theoretical labeled +2.00D power. The compensation power of the FCL prototypes was negatively correlated with the experimental 
therapeutic power $(r=-0.786, p=0.007)$; i.e.; in the prototypes we found that the higher was the absolute value of the compensation power, the lower was the therapeutic power of the lens.

When fitted in patients, topological data revealed that CLs were decentered towards temporal cornea, ranging from $0.39 \mathrm{~mm}$ to $1.05 \mathrm{~mm}$ (mean $0.7 \pm 0.19 \mathrm{~mm}$ ) whereas mean vertical displacement was $0.00 \pm 0.49 \mathrm{~mm}$ (ranging from $0.64 \mathrm{~mm}$ down to $1.38 \mathrm{~mm}$ up). The mean value of the pupil entrance diameter was $3.67 \pm 0.53 \mathrm{~mm}$ measured with the Keratron in the nacked eye. See Figure 3.

The mean values of the corneal asphericities along a $4 \mathrm{~mm}$ of semi-chord in the horizontal meridian were $-0.07 \pm 0.09$ and $-0.24 \pm 0.18$ for the temporal and nasal cornea respectively. No correlations were found between the amount of lens decentering and the asphericity of the cornea along temporal and nasal sides.

\section{Relative Peripheral Refractive Error}

Significant correlations were found between the corneal asphericity and vector components of the RPRE at several points along the horizontal meridian in the nacked eye. These values are reported in Table 1.

Baseline mean values of the RPRE for $M, J_{0}$ and $J_{45}$ are represented in Figures $4 A, 4 D$ and $4 G$, respectively. Figures 4B, 4E and 4H show the mean values for the same eyes wearing FCLs. The net effect of the lens is shown in Fig. 4C, 4F, and $4 \mathrm{I}$. In these plots, crosses were drawn for those positions where the myopic RPRE induced by the FCL is statistically significant. It can be seen that the mean myopic shift induced by FCLs increases with the eccentricity and becomes significant $(\mathrm{p}<0.05)$ at $10^{\circ}$ in the temporal retina (nasal visual field). Note that the effect of the FCLs on the refractive components was to produce an asymmetry from nasal to temporal visual 
fields (Figures 4B, 4C, 4F and 4I), reflecting the lens decentration to the temporal cornea. In fact, the effect of the lens on the spherical equivalent $M$ was almost uniform around the center of the lens, while, as expected, affects mainly the horizontal and vertical meridians for the $\mathrm{J}_{0}$ component and the same for the $\mathrm{J}_{45}$ component in oblique meridians for (see Figure $4 \mathrm{G}$ and Figure $4 \mathrm{H})$.

Figure 5A shows the spherical equivalent (M) along the horizontal meridian, both, at the baseline state and with the FCLs. An increase of the myopic shift was found with the FCLs at the temporal retina from $10^{\circ}$ to $30^{\circ}(\mathrm{p}<0.05)$.The $\mathrm{M}$ values of the RPRE computed by ray tracing in our previous work, ${ }^{11}$ on a myopic model eye $(-2,00 \mathrm{D})$ fitted with a FCL of $-2.00 \mathrm{D}$, having therapeutic zones $1.32 \mathrm{D}$ and decentered $3.67 \mathrm{~mm}$ horizontally are shown in the same figure. An excellent agreement can be observed between numerical and the experimental results. We found that the myopic shift (M) induced by the FCLs at $25^{\circ}$ and $30^{\circ}$ in the temporal field decreased with the lens decentration through the temporal side of the cornea $r=0.50(p=0.013)$ at $25^{\circ}$ and $r=0.54(p=0.006)$ at $30^{\circ}$. The tangential and sagittal power errors along the horizontal meridian are shown in Fig 5B and 5C. As can be seen the FCLs produce an increasing myopic shift in the $\mathrm{F}_{\mathrm{T}}$ curve, with a maximun value at $20^{\circ}$ of the temporal retina. Fs also reveals a myopic shift with the FCL, even though less markedly than $\mathrm{F}_{\mathrm{T}}$, but highly enough to move the sagittal foci to the front of the retina.

\section{Discussion}

Derived from experimental studies in animals, that found that the refractive error in the peripheral retina can regulate the eye growth, current successful treatments aimed to slow myopia progression, such as ortokeratology or multifocal contact lenses are intended to create a relative peripheral myopia. In most of the clinical studies, the study of the effect of the proposed 
solutions was restricted to the analysis of the RPRE, measured in terms of vector components M, $\mathrm{J}_{0}$ and $\mathrm{J}_{45}$ along the horizontal meridian, ${ }^{3,6}$ and only few include the vertical and oblique meridians. ${ }^{19-21}$

In this work we have presented the first clinical study in which the ability of a new design of CLs [10] to create a RPRE in the whole 2D visual field has been demonstrated. Taking into account that, on the one hand, the mean power at the treatment zones $(+1.32 \mathrm{D})$ of the manufactured lenses was lower than the theoretical labelled power (+2.00 D); and, on the other hand, that lens prototypes were decentered during the RPRE measurements an excellent agreement between the theoretical and experimental results has been obtained (see Fig.5A).

A negative correlation was also obtained between the compensation power of the prototypes and the power at the therapeutic zones, which means that high power minus lenses had less power in the therapeutic zones, than low power FCLs. This effect, that can be attributed to the spherical aberration, should be taken into account by manufacturers and prescribers, since as we have shown in our theoretical model, higher degrees of myopia should need higher treatment powers. In our design, this limitation could be easily solved since the treatment power this is a free parameter that affects the peripheral refractive error without compromising the central vision, at least for pupil diameters lower than $4.0 \mathrm{~mm} .^{10}$

For the nacked eye, we found that corneal asphericity along temporal and nasal semichords in the horizontal meridian was negatively correlated with the $\mathrm{M}$ component; but only for temporal cornea and nasal retina whereas for $\mathrm{J}_{45}$ and $\mathrm{J}_{0}$ the negative correlations where found between nasal cornea and temporal retina (Table 1). This is also in agreement with theoretical models, which assert that the more positive is Q, the more myopic is the peripheral refraction induced for $\mathrm{M}$ and $\mathrm{J}_{0}{ }^{22,23}$ Therefore, this fact should be considered by clinicians, because more prolate 
corneas might be expected to progress into more myopia. ${ }^{24,25}$ For the naked eye, the sample showed a relative peripheral myopia lower than $-0.50 \mathrm{D}$ for $\mathrm{M}$. We also found a trend for $\mathrm{J}_{0}$ to become more negative in both sides of the retina, whereas $\mathrm{J}_{45}$ becomes more negative with increasing eccentricity in the superior-nasal to inferior-temporal visual field. These results agree with those reported in previous studies that measured peripheral $\mathrm{M}$ and oblique astigmatism in myopes with a Hartmann-Shack aberrometer. ${ }^{26}$ On the other hand in our sample, the sagittal focus was hyperopic in the naked eye along the nasal visual field (Figure 5C), but became myopic with the FCLs. This result is an improvement of FCLs over the ortokeratology for myopia control, because in ortokeratology, especially in low myopes, this effect has not been

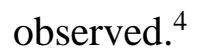

In conclusion, in this work we have experimentally validated ability of the FCLs to create a myopic RPRE in myopic eyes. Excellent agreement between the theoretical prediction and the experimental results was obtaneid. In fact, we also have confirmed that, considering both the imperfections in the manufacturing process of the prototypes, and the lens decentration, the theoretical model used in Ref. [10] can accurately be used to predict the lens effect in real eyes. The FCL design was intended to not interfere with the normal functioning of accommodation; i.e. to avoid any additional blur at near vision under photopic lighting conditions. ${ }^{10}$ This is also important because the role that accommodation plays in the myopia progression is still an issue to be resolved. ${ }^{27}$

On the other hand, the new two-dimensional representation of the RPRE employed to study the effect of the lens in different areas of the visual field, offered us further information about what happens in a wide area of the retina, especially considering the lens decentration. This means that this representation is convenient for increasing the knowledge about the changes in the 
astigmatism components induced by the lenses. For instance, we found that for the $\mathrm{J}_{45}$ component minor changes are induced by the lens that can only be appreciated in oblique meridians.

\section{Funding.}

This work was founded by Ministerio de Economía y Competitividad FEDER (Grant DPI201571256-R), and by Generalitat Valenciana (Grant PROMETEOII-2014-072), Spain.

\section{Declaration of interests:}

M. Rodríguez-Vallejo, J. A. Monsoriu and W. D. Furlan are inventors: ES Patent P201330862, relating to contact lens design: assigned to Universidad Politécnica de Valencia and Universitat de Valéncia.

D. Montagud and V. Ferrando report no conflicts of interest.

The authors alone are responsible for the content and writing of the paper.

\section{References}

1. Wolffsohn JS, Calossi A, Cho P, Gifford K, Jones L, Li M, et al. Global trends in myopia management attitudes and strategies in clinical practice. Cont Lens Anterior Eye. 2016;39:106-116.

2. Huang J, Wen D, Wang Q, McAlinden C, Flitcroft I, Chen H, et al. Efficacy comparison of 16 interventions for myopia control in children. Ophthalmology. 2016;123:697-708.

3. Walline JJ. Myopia control: A review. Eye Contact Lens. 2016;42:3-8.

4. González-Méijome JM, Faria-Ribeiro MA, Lopes-Ferreira DP, Fernandes P, Carracedo G, Queiros A. Changes in peripheral refractive profile after orthokeratology for different degrees of myopia. Curr Eye Res. 2015;41:199-207. 
5. Queirós A, Lopes-Ferreira D, González-Méijome JM. Astigmatic peripheral defocus with different contact lenses: review and meta-analysis. Curr Eye Res. 2016;Feb 2:1-11

6. González-Méijome JM, Peixoto-de-Matos SC, Faria-Ribeiro M, Lopes-Ferreira DP, Jorge J, Legerton J, et al. Strategies to regulate myopia progression with contact lenses: a review. Eye Contact Lens. 2016;42:24-34.

7. Atchison DA, Rosén R. The Possible Role of Peripheral Refraction in Development of Myopia. Optom Vis Sci. 2016;93:1042-1044.

8. Li S-M, Kang M-T, Wu S-S, Meng B, Sun Y-Y, Wei S-F, et al. Studies using concentric ring bifocal and peripheral add multifocal contact lenses to slow myopia progression in school-aged children: a meta-analysis. Ophthalmic Physiol Opt. 2017;37:51-59

9. Turnbull PR, Munro OJ, Phillips JR. Contact lens methods for clinical myopia control. Optom Vis Sci. 2016;93:1-7.

10. Rodríguez-Vallejo M, Benlloch J, Pons A, Monsoriu JA, Furlan WD. The effect of Fractal Contact Lenses on peripheral refraction in myopic model eyes. Curr Eye Res. 2014 21;39:1151-1160.

11. Charman WN. Keeping the world in focus: how might this be achieved? Optom Vis Sci. 2011;88:373-376.

12. Kee CS, Hung LF, Qiao-Grider Y, Roorda A, Smith EL. Effects of optically imposed astigmatism on emmetropization in infant monkeys. Invest Ophthalmol Vis Sci. 2004;45:1647-1659.

13. Chu CH, Kee CS. Effects of optically imposed astigmatism on early eye growth in chicks. PLoS One. 2015;10:e0117729.

14. Rodríguez-Vallejo M, Montagud D, Monsoriu JA, Furlan WD. On the power profiles of contact lenses measured with NIMO TR1504. J Optom. 2017;10:265-266.

15. Plainis S, Atchison DA, Charman WN. Power profiles of multifocal contact lenses and their interpretation. Optom Vis Sci. 2013;90:1066-1077.

16. Calossi A. Corneal asphericity and spherical aberration. J Refract Surg. 2007;23:505-514.

17. Lopes-Ferreira DP, Neves HIF, Faria-Ribeiro M, Queirós A, Fernandes PRB, González- 
Méijome JM. Peripheral refraction with eye and head rotation with contact lenses. Cont Lens Anterior Eye; 38,104-109;

18. Thibos LN, Wheeler W, Horner D. Power vectors: an application of Fourier analysis to the description and statistical analysis of refractive error. Optom Vis Sci. 1997 Jun;74:367-375.

19. Ehsaei A, Mallen EA, Chisholm CM, Pacey IE. Cross-sectional sample of peripheral refraction in four meridians in myopes and emmetropes. Invest Ophthalmol Vis Sci. 2011;52:7574-7585.

20. Osuagwu UL, Suheimat M, Atchison DA. Peripheral aberrations in adult hyperopes, emmetropes and myopes. Ophthalmic Physiol Opt. 2017;37:151-159.

21. Verkicharla PK, Suheimat M, Schmid KL, Atchison DA. Peripheral Refraction, Peripheral Eye Length, and Retinal Shape in Myopia. Optom Vis Sci. 2016;93:1072-1078.

22. Atchison DA. Optical models for human myopic eyes. Vis Res. 2006;46:2236-2250.

23. He JC. Theoretical model of the contributions of corneal asphericity and anterior chamber depth to peripheral wavefront aberrations. Ophthalmic Physiol Opt. 2014;34:321-330.

24. Horner DG, Soni PS, Vyas N, Himebaugh NL. Longitudinal changes in corneal asphericity in myopia. Optom Vis Sci. 2000;77:198-203.

25. Hiraoka T, Kotsuka J, Kakita T, Okamoto F, Oshika T. Relationship between higher-order wavefront aberrations and natural progression of myopia in schoolchildren. Sci Rep.; 2017;7:7876

26. Osuagwu UL, Suheimat M, Atchison DA. Mirror Symmetry of Peripheral Monochromatic Aberrations in Fellow Eyes of Isomyopes and Anisomyopes. Investig Opthalmology Vis Sci. 2016;57:3422-3427.

27. Schaeffel F, Wildsoet C. Can the retina alone detect the sign of defocus? Ophthalmic Physiol Opt. 2013;33:362-367. 


\section{Tables}

Table 1. Correlations between Relative Peripheral Refractive Error (RPRE) vector components and asphericity at the Temporal or Nasal semi-chord of the cornea from the normal vertex to 4 mm of radial position in the nacked eye. Only significant values are included.

\begin{tabular}{ccccl}
\multicolumn{2}{c}{ Retinal Area $\left(^{\circ}\right)$} & $\begin{array}{c}\text { RPRE (D) } \\
\text { Mean } \pm \text { SD }\end{array}$ & Corneal side & Pearson $\mathrm{r}$ \\
\hline $\mathrm{M}$ & & & & \\
& $-25(\mathrm{NR})$ & $-0.22 \pm 0.47$ & Temporal & $-0.452, \mathrm{p}=0.040$ \\
& $-15(\mathrm{NR})$ & $-0.21 \pm 0.40$ & Temporal & $-0.526, \mathrm{p}=0.014$ \\
$\mathrm{~J} 0$ & $-10(\mathrm{NR})$ & $-0.27 \pm 0.29$ & Temporal & $-0.436, \mathrm{p}=0.048$ \\
& & & & \\
& $+25(\mathrm{TR})$ & $-0.82 \pm 0.29$ & Nasal & $-0.572, \mathrm{p}=0.007$ \\
& $+20(\mathrm{TR})$ & $-0.56 \pm 0.22$ & Nasal & $-0.562, \mathrm{p}=0.008$ \\
$\mathrm{~J} 45$ & $+10(\mathrm{TR})$ & $-0.1 \pm 0.2$ & Nasal & $-0.505, \mathrm{p}=0.019$ \\
& & & & \\
& $+30(\mathrm{TR})$ & $0.11 \pm 0.25$ & Nasal & $-0.581, \mathrm{p}=0.006$ \\
& $+20(\mathrm{TR})$ & $0.05 \pm 0.15$ & Nasal & $-0.465, \mathrm{p}=0.033$ \\
& $+10(\mathrm{TR})$ & $-0.01 \pm 0.09$ & Nasal & $-0.478, \mathrm{p}=0.028$ \\
& $+5(\mathrm{TR})$ & $0.01 \pm 0.09$ & Nasal & $-0.467, \mathrm{p}=0.033$ \\
\hline
\end{tabular}

$\mathrm{NR}=$ Nasal retina; $\mathrm{TR}=$ Temporal retina. 


\section{Captions}

Figure 1. A) Theoretical bifocal profile of the FCL lens curvature B) Measured zones in a constructed FCL with mean far power -3.5 D. Vertical lines are the limits of the zones detected by the custom software (see Ref. 14). C) Profile of the treatment power across the lens obtained by substracting the base (far) power to the curve in Fig. B). D). Radial variation of the base and treatment powers fitted to a parabolic radial profile reflecting the spherical aberration of the lens.

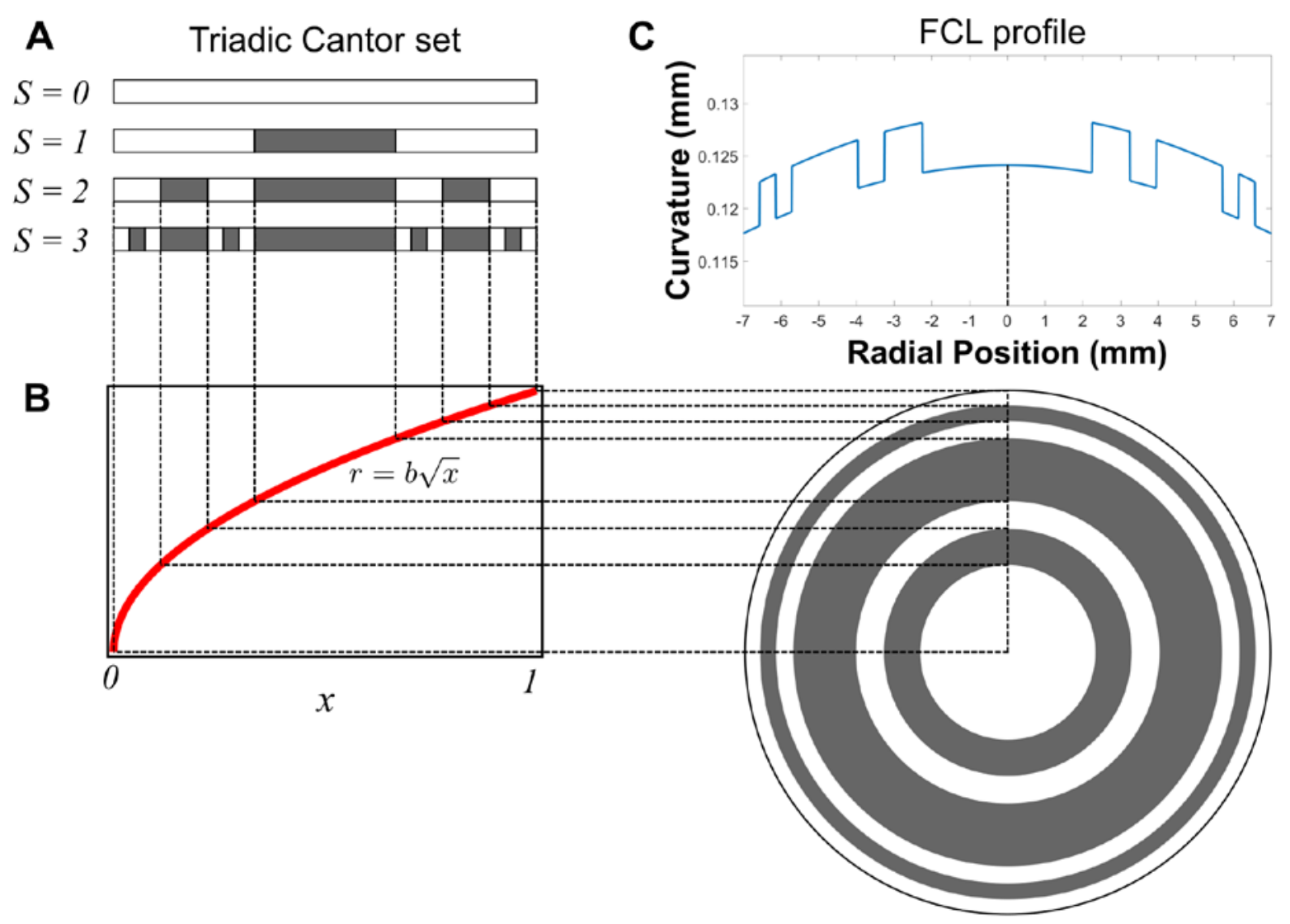


Figure 2. Schematic representation of the RPRE measurement process with WAM-5500. The circles represent the discrete points measured with the eye rotation method.

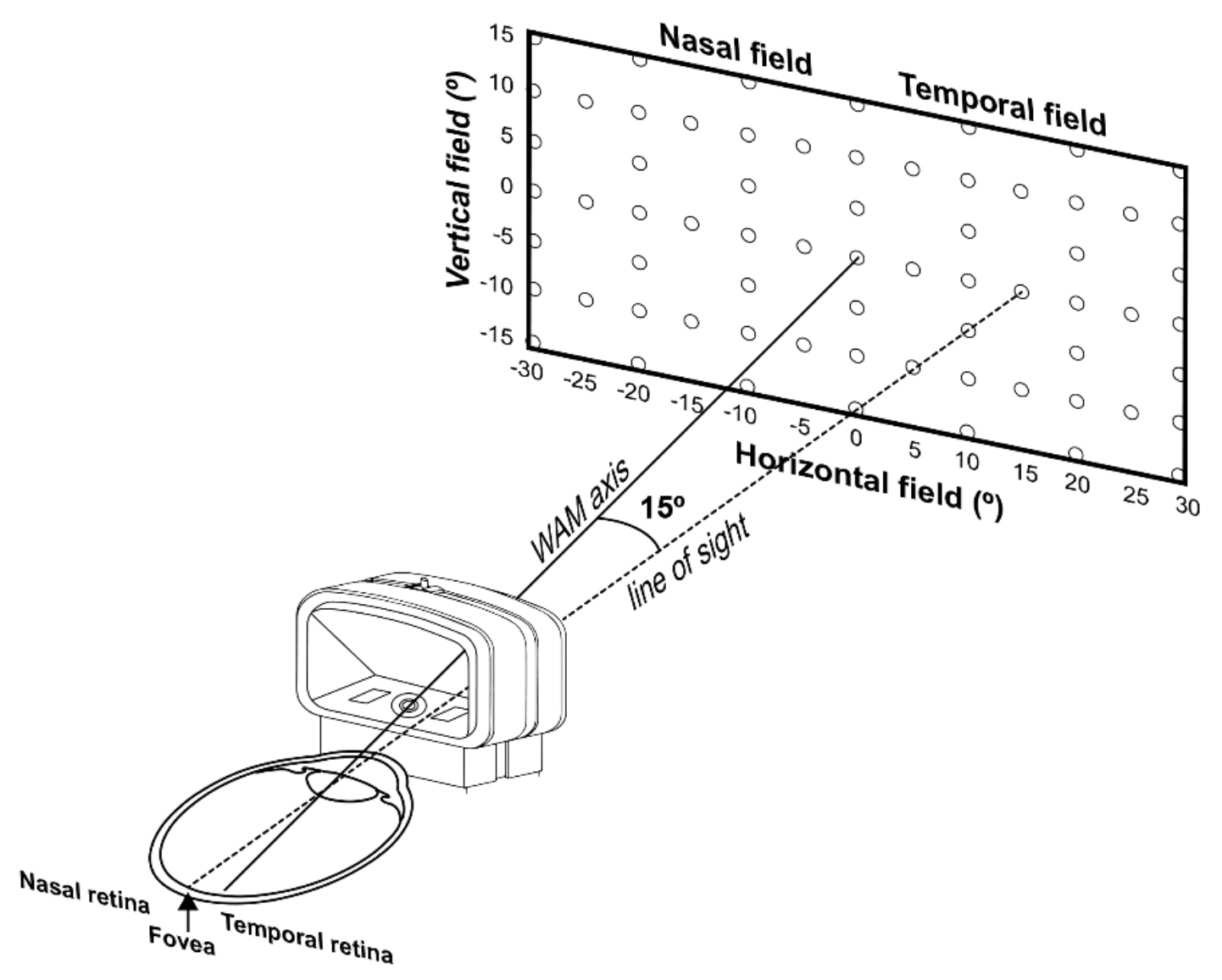


Figure 3. Topographic image of the FCL fitted in a patient's eye. Black and white crosses indicate the centers of the pupil and the lens respectively. In this case a decentration of $0.57 \mathrm{~mm}$ was measured.

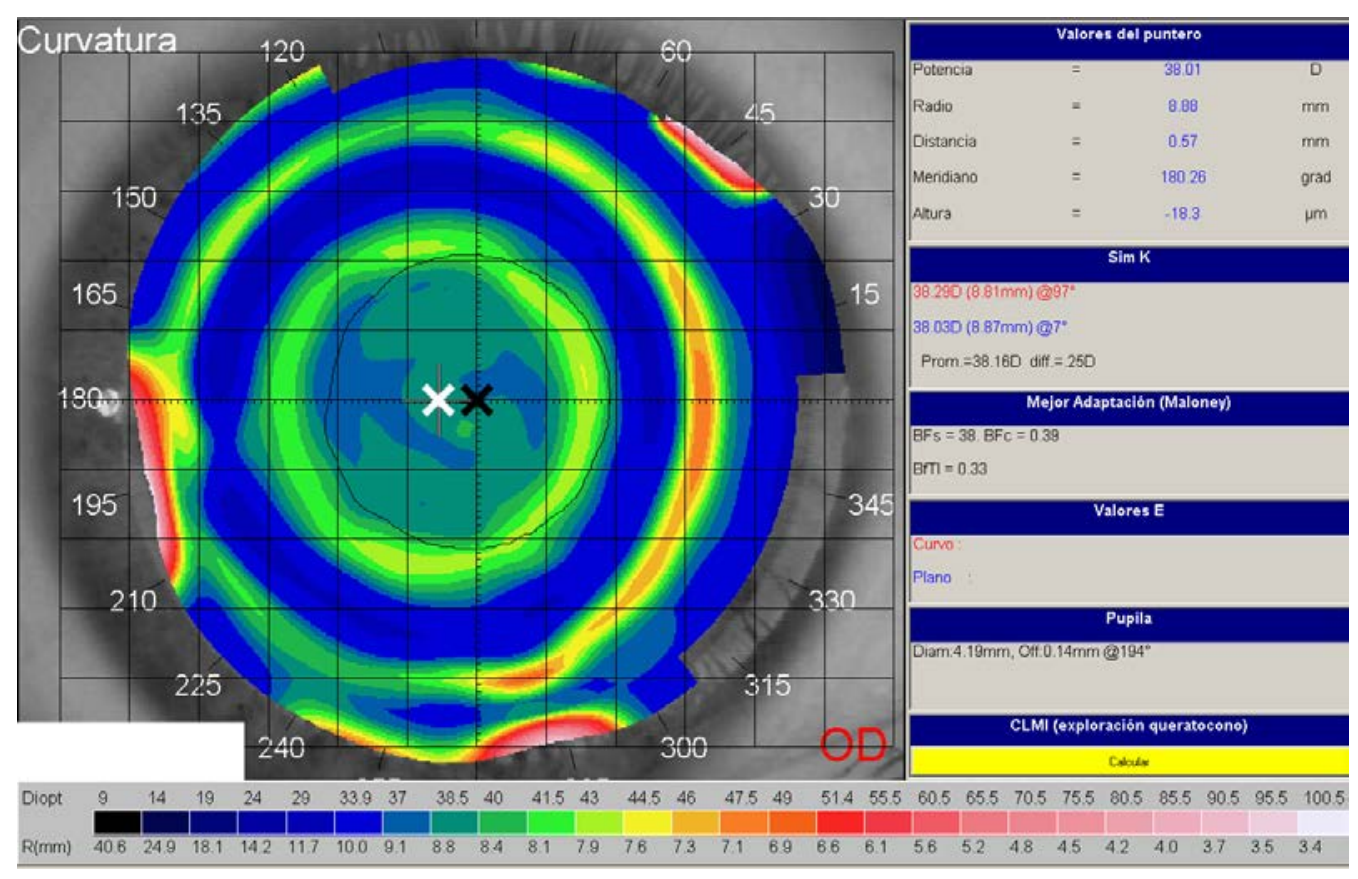


Figure 4. Mean values of the relative peripheral refractive error (RPRE) across the visual field for the vector components $\mathrm{M}, \mathrm{J}_{0}$ and $\mathrm{J}_{45}$, at the baseline state (left); with FCLs fitted in the eye (centre) and the difference (right). Crosses in the right column indicate field positions (see Fig. 2) where the myopic RPRE induced by the FCL is statistically significant $(\mathrm{P}<0.05)$.

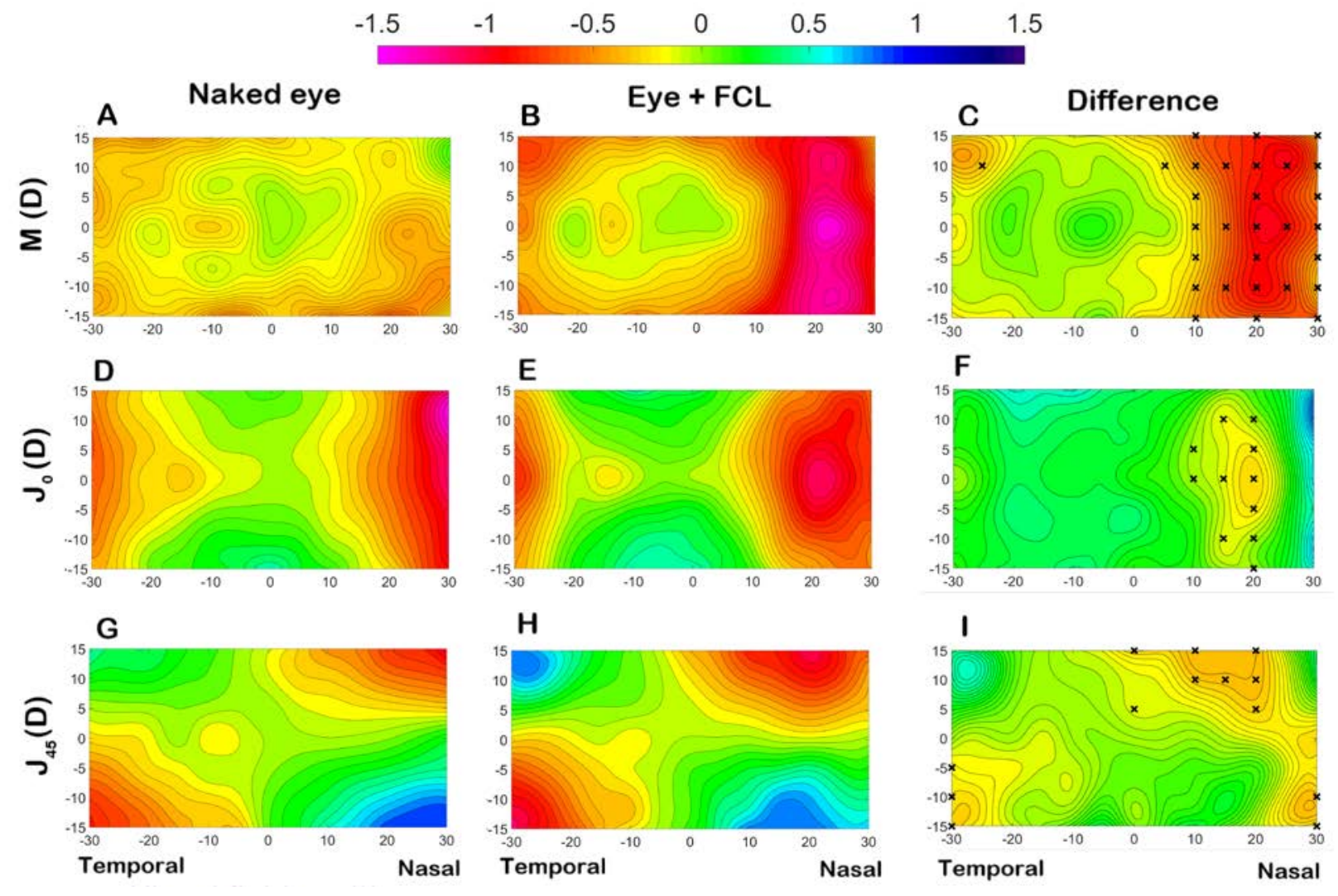


Figure 5. (A) Mean values of the spherical equivalent M (RPRE) along the horizontal visual field in the nacked eye (Baseline) and with Fractal Contact Lenses (FCL) The black symbols and dashed line represent the theoretical values computed by ray tracing (see the main text for details). (B) Tangential and (C) Sagittal powers along the horizontal retina in the baseline state and with FCLs. An asterisk over each eccentricity was represented to describe significant differences between baseline and with FCLs $(\mathrm{p}<0.05)$.
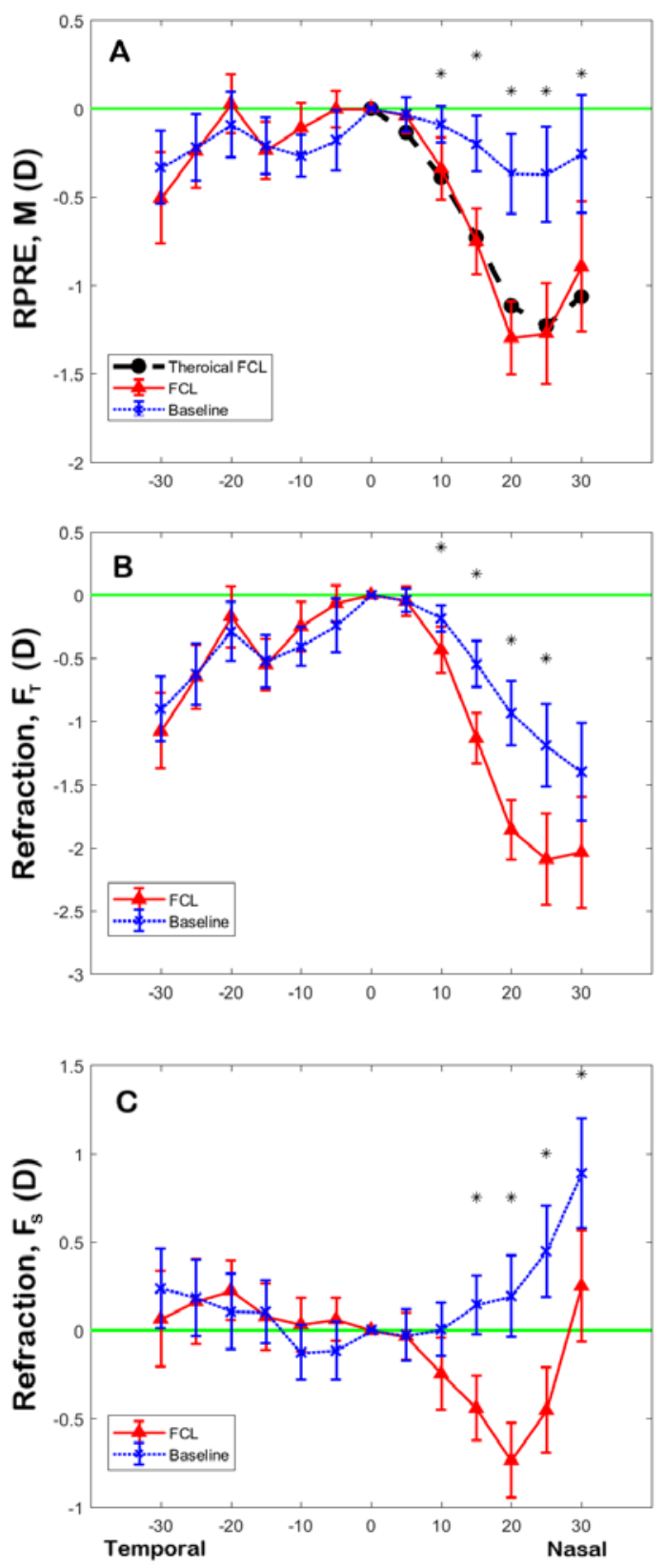